\title{
BMJ Open From 'screen time' to the digital level of analysis: protocol for a scoping review of digital media use in children and adolescents
}

\author{
Dillon Thomas Browne (D) , ${ }^{1}$ Shealyn May, ${ }^{1}$ Pamela Hurst-Della Pietra, ${ }^{2}$ \\ Dimitri Christakis, ${ }^{3}$ Tracy Asamoah, ${ }^{4}$ Lauren Hale, ${ }^{5}$ Katia Delrahim-Howlett, ${ }^{6}$ \\ Jennifer A Emond, ${ }^{7}$ Alexander G Fiks, ${ }^{8}$ Sheri Madigan, ${ }^{9}$ Heather Prime, ${ }^{10}$ \\ Greg Perlman, ${ }^{11}$ Hans-Jürgen Rumpf, ${ }^{12}$ Darcy Thompson, ${ }^{13}$ Stephen Uzzo, ${ }^{14}$ \\ Jackie Stapleton, ${ }^{15}$ Ross Neville, ${ }^{16}$ the Media Impact Screening Toolkit Workgroup \\ of Children and Screens: Institute of Digital Media and Child Development
}

To cite: Browne DT, May S, Hurst-Della Pietra P, et al. From 'screen time' to the digital level of analysis: protocol for a scoping review of digital media use in children and adolescents. BMJ Open 2019;9:e032184. doi:10.1136/ bmjopen-2019-032184

- Prepublication history and additional material for this paper are available online. To view these files, please visit the journal online (http://dx.doi org/10.1136/bmjopen-2019032184).

Received 06 June 2019 Revised 02 October 2019 Accepted 16 October 2019

Check for updates

(c) Author(s) (or their employer(s)) 2019. Re-use permitted under CC BY-NC. No commercial re-use. See rights and permissions. Published by BMJ.

For numbered affiliations see end of article.

Correspondence to Dr Dillon Thomas Browne; dillon.browne@uwaterloo.ca

\section{ABSTRACT}

Introduction Research on the relationship between digital media exposure and child development is complex, inconsistent and fraught with debate. A highlighted area of inadequacy surrounds the methodological limitations of measuring digital media use for both researchers and clinicians, alike. This protocol aims to (1) identify core concepts in the area of screen time and digital media use in children and adolescents (2) map existing research paradigms and screening/measurement tools that serve to underpin and operationalise core concepts and (3) provide an initial step in integrating these findings into a consolidated screening toolkit. It is expected this enterprise will help advance research and clinical evaluation in fields concerned with digital media use, namely medicine, child development and the social sciences.

Methods and analysis The planned scoping review will search relevant electronic databases, including Ovid MEDLINE, PsycINF0 and Scopus, in addition to grey literature. All empirical investigations and presentation of original research will be considered, and measurement/screening tools for digital media usage in children and adolescents will be identified and reported on. Two reviewers will pilot test the screening criteria, and data extraction forms prior to independently screening all relevant literature and extracting the data. A three-stage synthesis process will be used to map the existent measurement and screening tools for digital media usage in children and adolescents.

Ethics and dissemination There are no ethical considerations for this scoping review. Plans for dissemination include publication in a top-tier, open-access journal, public presentations and conference proceedings. Presentation of the full scoping review has been accepted to the American Academy of Child \& Adolescent Psychiatry 66th Annual Meeting.

\section{INTRODUCTION}

\section{Rationale}

There has been growth in scientific research on the potential developmental effects of screen time exposure and digital media usage
Strengths and limitations of this study

- Our scoping review will be comprehensive, including searches from multiple databases and grey literature, notably institutional reports and guidelines.

- Our scoping review has a novel approach, with a focus on the source's methodology (ie, tools of digital media use measurement) for data extraction.

- Our scoping review will be guided by the methods outlined in the Joanna Briggs Institute Reviewer's Manual and will adhere to the Preferred Reporting Items for Systematic Reviews and Meta-Analyses extension for scoping reviews reporting standards, in addition to recommendations for grey literature (eg, Cochrane Handbook).

- A less detailed analysis of project-specific interventions and outcomes is provided.

- Due to the magnitude of sources in the field, only empirical investigations or original research targeting the development and testing of a measurement or screening tool for digital media interaction will be included in our review. No theoretical sources will be included, and the sources must be published in English published in the last 5 years.

for children and adolescents. Research has determined that the relationship between digital media exposure and child development is complex, with some findings supporting negative consequences, others indicating positive outcomes, and some studies showing little to no association. ${ }^{1-3}$ Studies on neurocognitive or socioemotional developmental effects consistently highlight the need for a valid and reliable protocol to efficiently and comprehensively measure screen time and digital media usage patterns in young people and families. ${ }^{4-6}$ Indeed, the extant scientific literature and popular commentary are 
fraught with debate, highlighting the challenges, inconsistencies and inadequacies pertaining to the definition of constructs, in addition to methodological limitations in measuring media use for researchers and clinicians, alike. Clearly, the question 'what is 'screen time' and 'digital media use' and how do we measure them?' emerges as an obvious, yet surprisingly unanswered area for consideration.

Critics have raised concerns around efforts to document associations between the 'amount' or 'duration' of time spent using devices and child outcomes as imprecise and abstruse, yet exacting measurement alternatives are not widely available or employed. ${ }^{78}$ Similarly, clinicians who work with distressed caregivers and struggling children are requesting measurement and screening tools to use in their practices that can ascertain these nuances while also providing timely clinical solutions for busy providers. In light of these challenges, the planned protocol for a scoping review aims to outline a plan to review and synthesise the literature in a way that will clarify conceptual, definitional and methodological challenges in digital media use research with children, particularly in the area of developmental science, psychology/ psychiatry and paediatrics. This will serve as an initial step in a larger initiative to create a suite of state-of-the-art clinical tools that will be of use to researchers and clinicians who are interested in this phenomenon.

The proposed scoping review represents a collective effort of over 30 developmental scientists, psychiatrists, paediatricians, psychologists, social workers, caregivers and other stakeholders who are invested in advancing research and practice with children and youth in a world that has become increasingly digitally mediated. This collective effort grew out of a professional meeting, namely, the second Digital Media and Developing Minds Congress at Cold Spring Harbor Laboratory, Long Island, New York (15-18 October 2018), hosted by the Children and Screens: Institute for Digital Media and Developing Minds, a non-profit organisation. A workgroup was formed (ie, the Media Impact Screening Toolkit Workgroup of Children and Screens: Institute for Digital Media and Developing Minds), emerging from a conference exercise where participants were asked to discuss and present on issues relating to the measurement of screen time and digital media use. This exercise clearly emphasised the importance of and opportunity to form a workgroup whose mandate it was to champion this initiative. Additionally, the horizon goal of creating a toolkit of high-quality, cutting-edge and scientifically sound tools to measure and screen digital media usage in children was identified. However, this enterprise, in and of itself, proved to be very complex and challenging. Who would be the 'respondent' for such measures? Would we also harness the power of automatic data capture? Should we partner with Big Tech to request existing data that is collected on all device users? What type of devices? And is all screen time really the same, anyway? Should we be tracking hours, content or both?
From these conversations, an initial step in our workgroup's vision became clear. We needed to conduct a scoping review, the purpose of which is to provide clarity, insight and a conceptual basis on which to form the downstream measurement goals of our group. The scoping review would provide a transparent and documented forum, whereby the sum of these important questions are presented, integrated and distilled into important dimensions for consideration in the world of digital media use research and practice. Moreover, with the speed of innovation supporting changes in technology and devices, the importance of having a dynamic, responsive and living entity (ie, our workgroup) was highlighted, in addition to the development of a reliable, comprehensive and adaptable media interaction screen toolkit. The scientific process surrounding the development, validation and dissemination of the NIH Toolbox measures for neurocognitive and socioemotional functioning in youth was cited as an exemplar, and representation from the developers (from healthmeasures.net) on our workgroup was solicited. To eventually design a media interaction screening toolkit for clinicians and researchers, it was clear that we had to begin with the question: "what has been done?" Thus, we proposed a scoping review that would organise important dimensions of consideration in the field while also providing a review of many existing measurement tools (eg, amount of digital media use), screening tools (eg, screening for problematic use) and paradigms for media interaction and screen time. Given the nascent and disparate nature of the field, a scoping review was determined to be the optimal method of knowledge synthesis, versus a systematic review, meta-analysis or otherwise. From the scoping review, we hope to identify important sources of variation in screen time and digital media use (eg, frequency, intensity, time, timing and type of use) that our proposed measurement system will be sensitive enough to capture. Any comprehensive screening toolkit for screen time and digital media use will certainly build on the already large and disparate body of research that has been conducted on this topic.

\section{Objectives}

The present scoping review aims to ${ }^{1}$ identify core concepts in the area of screen time and digital media use in children and adolescents, ${ }^{2}$ map existing research paradigms and measurement/screening tools that serve to underpin and operationalise these key dimensions and $^{3}$ integrate these findings into a preliminary consolidated screening toolkit, to be further developed using systematic reviews and/or meta-analyses and validation testing. In concert with the broader objectives of our workgroup, findings from the scoping review will inform a large-scale psychometric initiative that seeks to develop a reliable, valid, utilitarian and widely employed suite of instruments that can be deployed by clinicians and scientists to screen, monitor and measure media habits in children and adolescents. It is our expectation that these instruments will help advance the field of digital 
media research while also addressing the concerns of researchers who call existing screen time research into question. We also anticipate that this scoping review will illustrate the need to move beyond considering 'screen time' as a simple exposure variable to theoretically integrating the 'digital level of analysis' into models of human development; that is, consider digital media interaction as a 'scale into which behaviour or the brain can be represented. ${ }^{10}$ On completion of the scoping review, next steps for the project will include additional reviews (ie, systematic reviews or meta-analyses) to further develop the consolidated screening toolkit, followed by validation testing.

\section{METHODS AND ANALYSIS \\ Identification of relevant studies}

The scoping review will include studies in which direct screening of media interaction of persons aged 0-25 years is presented. A search for relevant studies will be conducted using the following databases: Ovid MEDLINE, PsycINFO and Scopus. Comprehensive search strategies consisting of author keywords and subject headings have been developed in consultation and collaboration with liaison librarians (also known as 'Information Specialists') with specialisations in psychology and public health. Results will be limited to English language and published within the last 5 years (ie, 1 March 2014 to 2 March 2019). We considered starting the search in 2007 (iPhone release year); however, this yielded too many results to be feasible to screen for this scoping review. Also, we are most interested in the measurement of device use in the present technological landscape, so we do not feel that this will bias or systematically alter our conclusions. All literature searches will be conducted by a librarian at the University of Waterloo. Please refer to online supplementary material I (online) for the MEDLINE search strategy. Further techniques to identify relevant studies will include contacting knowledge experts and reviewing the references of included studies. A thorough search of organisation reports in the grey literature will also be conducted, consistent with guidelines from the Cochrane Handbook, Centre for Reviews and Dissemination and the Canadian Agency for Drugs and Technology in Health 'Grey Matters' guidelines. All bibliographic information will be amalgamated and stored using a citation management software, namely Endnote. This scoping review will be conducted from July 2019 until January 2020 (approximately).

\section{Study selection}

Titles and abstracts will be reviewed independently by two pretrained reviewers and marked as 'include', 'exclude' or 'unsure' based on the selection criteria using Covidence. Discrepancies will be resolved by a third reviewer based on an independent review of the source and feedback from the two original reviewers. Full text articles will be retrieved for studies deemed as either 'included' or 'unsure' and will be reviewed independently and in duplicate to ensure inclusion based on adherence to the selection criteria. All reviewers will use a screening form developed for this review to screen each study at each stage of the selection process. Due to the complexity of digital media interaction, any sources that remain identified as 'uncertain' after full text screening will be discussed among the entire team until a consensus is determined. Please see online supplementary material II for selection criteria.

\section{Data extraction}

All studies deemed as 'included' will be reviewed independently by two reviewers using a data extraction form created for this review. The data extraction form is presented in online supplementary material III. This data extraction form will be pilot tested on 20 articles to ensure high inter-rater reliability and establish functionality of the form. Any necessary changes will be made following this pilot testing prior to extracting data from all included articles. The following data will be extracted (where available): title, author(s), year of publication, country of origin, publication type, aims/purposes, study population, sample size, study setting, study methodology, digital media source(s), screen type(s), definition of media interaction, measurement/screening tool name, measurement type, targeted populations, informant type, statements of measurement/screening tool reliability and statements of measurement/screening tool validity. Methodological quality (including study bias) will be assessed at this time based on the series of judgements proposed by Cochrane. ${ }^{11}$ Each area of risk will be judged as 'low risk', 'high risk' or 'unclear'. Any studies deemed biased will be considered with caution and noted in the data synthesis stage. Risk assessment and data charting will be completed using Covidence. Once all studies have been reviewed, extracted data will be exported into SPSS for data analysis.

\section{Data synthesis}

Results of the review will be presented using the following strategies:

1. A chart providing a numerical overview of the amount, type and categorisation of the included studies.

2. An overview table containing all included studies sorted by sample population age listing study type, media type, measurement/screening tool name, measurement type and psychometric properties of the measurement/screening tool.

3. A narrative synthesis and mapping of the included studies to establish the extent of the literature.

This presentation of the results will outline and categorise measurement and screening tools from the included studies, which can be used to understand the nature and extent of the existent instruments for measuring media interaction with child populations. Meta-analysis of quantitative study results will not be conducted at this time, as 
this is beyond the objectives of this review and the scoping review methodology.

\section{Patient and public involvement}

No patients were involved in the conceptualisation or design of this research protocol.

\section{Ethics and dissemination}

There are no ethical considerations for this scoping review.

Following the completion of the scoping review, a stakeholder meeting of the Media Impact Screening Toolkit workgroup will be held to discuss the implications of our findings and to finalise our dissemination strategy. A summary of the results will be published in a top-tier, open-access journal and will be shared through numerous online resources, including the Children and Screens: Institute for Digital Media and Developing Minds website. Presentation of the full scoping review has been accepted to the American Academy of Child \& Adolescent Psychiatry 66th Annual Meeting. Finally, authors and team members will be encouraged to share the results among their networks via professional websites and social media accounts to encourage a broad dissemination of the findings.

\section{Author affiliations}

${ }^{1}$ Psychology, University of Waterloo, Waterloo, Ontario, Canada

${ }^{2}$ School of Health Technology and Management, Stony Brook University, Stony Brook, United States

${ }^{3}$ University of Washington, Seattle, Washington, USA

${ }^{4}$ American Academy of Child and Adolescent Psychiatry, Washington, DC, USA

${ }^{5}$ Department of Preventive Medicine, State University of New York, Stony Brook,

Stony Brook, New York, USA

${ }^{6}$ National Institute on Drug Abuse, North Bethesda, Maryland, USA

${ }^{7}$ Biomedical Data Science, Dartmouth College, Lebanon, United States

${ }^{8}$ University of Pennsylvania Perelman School of Medicine, Philadelphia,

Pennsylvania, USA

${ }^{9}$ Psychology, University of Calgary, Calgary, Alberta, Canada

${ }^{10}$ Offord Centre for Child Studies, McMaster University, Hamilton, Ontario, Canada

${ }^{11}$ Renaissance School of Medicine, Stony Brook University, Stony Brook, New York, USA

${ }^{12}$ University of Lübeck Institute of the History of Medicine and Science Research, Lubeck, Schleswig-Holstein, Germany

${ }^{13}$ University of Colorado Denver School of Medicine, Aurora, Colorado, USA

${ }^{14}$ New York Hall of Science, Flushing, New York, USA

${ }^{15}$ Information Services and Resources, University of Waterloo, Waterloo, Ontario, Canada

${ }^{16}$ Physiotherapy and Sport Science, University College Dublin, Dublin, Ireland

Collaborators Member of the workgroup in addition to the authors listed above: Rachel Barr, Daphne Bavelier, Courtney K. Blackwell, Florence Breslin, Joanne Broder, Katherine Cost, Zsolt Demetrovics, Bernard Fuemmeler, John Hutton, Diane Kim, Heather Kirkorian, Monique LeBourgeois, Jessica Mendoza, Martin Paulus,
Jaysree Roberts, Thomas Robinson, Cris Rowan, Oren Shefet, Tim Smith, Rachel Waxman, and Paul Weigle. Authors would like to thank Juliette Givelas, Angelina Cleroux, Jackson Smith, Laura Colucci, Ben Southern, Heera Elize Sen, Julianna Lu and volunteer research assistants in the Whole-Family Lab for helping with study coding.

Contributors DTB and PH-DP obtained funding, conceptualised the research and edited the protocol. SSM conceptualised the research and drafted and edited the protocol. JS drafted the protocol search strategy and edited the protocol. TA, DAC, LH, KD-H, JAE, AF, SM, GP, HP, H-JR, DT, SU and RN conceptualised the research and edited the protocol.

Funding This work was supported by Children and Screens: Institute of Digital Media and Child Development (no applicable grant number). This funder, represented by Dr PH-DP, was involved in the design of the study protocol, the writing of the protocol, and the decision to submit the protocol for publication.

Competing interests None declared.

Patient consent for publication Not required.

Provenance and peer review Not commissioned; externally peer reviewed.

Open access This is an open access article distributed in accordance with the Creative Commons Attribution Non Commercial (CC BY-NC 4.0) license, which permits others to distribute, remix, adapt, build upon this work non-commercially, and license their derivative works on different terms, provided the original work is properly cited, appropriate credit is given, any changes made indicated, and the use is non-commercial. See: http://creativecommons.org/licenses/by-nc/4.0/.

ORCID iD

Dillon Thomas Browne http://orcid.org/0000-0002-7445-6604

\section{REFERENCES}

1 Barr R. Growing up in the digital age: early learning and family media ecology. Curr Dir Psychol Sci 2019;28:341-6.

2 Reid Chassiakos YL, Radesky J, Christakis D, et al. Children and adolescents and digital media. Pediatrics 2016;138:e20162593.

3 Kostyrka-Allchorne K, Cooper NR, Simpson A. The relationship between television exposure and children's cognition and behaviour: A systematic review. Developmental Review 2017;44:19-58.

4 King DL, Haagsma MC, Delfabbro PH, et al. Toward a consensus definition of pathological video-gaming: a systematic review of psychometric assessment tools. Clin Psychol Rev 2013;33:331-42.

5 Rich M, Bickham DS, Shrier LA. Measuring youth media exposure: a multimodal method for investigating the influence of media on digital natives. American Behavioural Scientist 2015;59:1736-54

6 Laconi S, Rodgers RF, Chabrol H. The measurement of Internet addiction: a critical review of existing scales and their psychometric properties. Comput Human Behav 2014;41:190-202.

7 de Vreese $\mathrm{CH}$, Neijens P. Measuring media exposure in a changing communications environment. Commun Methods Meas 2016;10:69-80.

8 Vandewater EA, Lee SJ. Measuring children's media use in the digital age: Issues and challenges. American Behavioural Scientist 2009;52:1152-76.

9 Cicchetti D. A multiple-levels-of-analysis perspective on research in development and psychopathology. In: Child and adolescent psychopathology, 2008: 27-57.

10 Masten AS, Cicchetti D. Developmental cascades. Dev Psychopathol 2010;22:491-5

11 Higgins JP, Altman DG. Assessing risk of bias in included studies. In: Cochrane handbook for systematic reviews of interventions: cochrane book series, 2008: 187-241. 